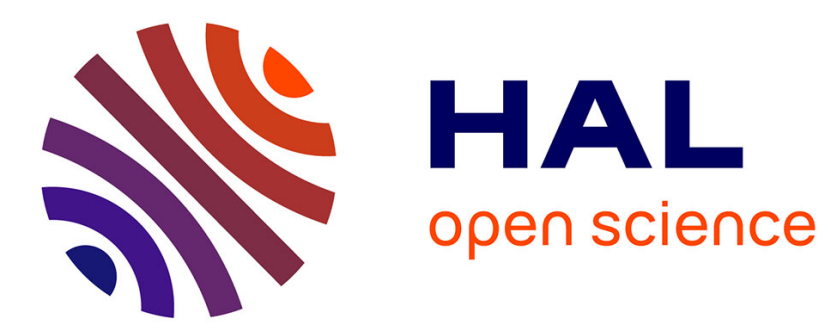

\title{
Mesure de rugosité par interférométrie acoustique et corrélation de speckles
}

C. Gazanhes, A. Calaora, R. Condat

\section{To cite this version:}

C. Gazanhes, A. Calaora, R. Condat. Mesure de rugosité par interférométrie acoustique et corrélation de speckles. Revue de Physique Appliquée, 1980, 15 (10), pp.1553-1561. 10.1051/rphysap:0198000150100155300 . jpa-00244880

\section{HAL Id: jpa-00244880 https://hal.science/jpa-00244880}

Submitted on 1 Jan 1980

HAL is a multi-disciplinary open access archive for the deposit and dissemination of scientific research documents, whether they are published or not. The documents may come from teaching and research institutions in France or abroad, or from public or private research centers.
L'archive ouverte pluridisciplinaire HAL, est destinée au dépôt et à la diffusion de documents scientifiques de niveau recherche, publiés ou non, émanant des établissements d'enseignement et de recherche français ou étrangers, des laboratoires publics ou privés. 


\title{
Mesure de rugosité par interférométrie acoustique et corrélation de speckles
}

\author{
C. Gazanhes, A. Calaora et R. Condat \\ Département d'Acoustique, C.N.R.S., 31 chemin Joseph-Aiguier, 13274 Marseille Cedex 2, France
}

(Reçu le 4 avril 1980, révisé le 19 juin 1980, accepté le 23 juin 1980)

\begin{abstract}
Résumé. - La rugosité d'une surface peut être déterminée par la corrélation de speckles acoustiques. On éclaire la surface à étudier sous deux incidences différentes au moyen de deux sources ultrasonores cohérentes. Les deux ondes diffusées (les speckles acoustiques) par la surface sont corrélées. La corrélation dépend des angles d'incidence, de la longueur d'onde ultrasonore et de l'écart-type des rugosités. Après avoir rappelé les principaux résultats relatifs à la corrélation des speckles, on décrit un interféromètre acoustique qui permet de réaliser cette corrélation. On étudie différentes rugosités de surface de béton $(0,025 \mathrm{~mm}<\sigma<2,39 \mathrm{~mm})$ avec une fréquence ultrasonore de $1 \mathrm{MHz}$. Les rugosités sont supposées normalement distribuées.
\end{abstract}

\begin{abstract}
Measurement of surface roughness can be achieved by means of acoustical speckle correlation. The same rough surface is illuminated by two coherent ultrasonic waves, under two different angles of incidence. The two scattered waves (the acoustical speckle) are correlated. The correlation depends on the standard deviation of the surface roughness, the angles of incidence and the ultrasonic wave lengh. Properties of speckle correlation are reviewed. An acoustical interferometer constructed on the basis of using speckle correlation is described. With this instrument and for normally distributed surfaces, an experimental investigation on surface roughness is conducted systematically for various concrete surface roughness $(0.025 \mathrm{~mm}<\sigma<2.39 \mathrm{~mm})$ with an ultrasonic frequency of $1 \mathrm{MHz}$.
\end{abstract}

1. Introduction. - Les études de rugosité de surface par voie acoustique reposent en général sur l'analyse des variations, en fonction de l'incidence et de la rugosité, de l'intensité ultrasonore moyenne rétrodiffusée. L'analyse s'effectue facilement en temps réel si la surface moyenne est fluctuante comme par exemple la surface de la mer [1]. Elle est plus longue et délicate dans le cas de surfaces solides peu rugueuses [2]. Cette dernière méthode est difficile à mettre en œuvre pour l'étude de surfaces rugueuses immergées en milieu marin [3], telles les surfaces de béton des ouvrages d'art, la surface des sédiments marins [4], etc. Pour ces surfaces qui possèdent une rugosité moyenne et forte, on a transposé à l'acoustique les méthodes optiques basées sur la corrélation des speckles [5]. Lorsqu'un objet diffusant (surface rugueuse) est éclairé par un laser, c'est-à-dire par une source émettant une lumière monochromatique spatialement et temporellement cohérente, tous les points de l'objet diffusent des vibrations lumineuses cohérentes donc capables d'interférer. En observant l'image de l'objet, on remarque une structure très fine formée de petits points lumineux. Cette structure granulaire est un speckle dû aux interférences des vibrations envoyées par les différents points de l'objet dans le plan image [6]. Les mêmes phénomènes existent en acoustique et plus particulièrement en haute fréquence avec les ondes ultrasonores. La figure 1 montre un exemple de speckle acoustique obtenu après réflexion d'une onde plane ultrasonore de $1 \mathrm{MHz}$ sur des surfaces rugueuses [7].

On montre que l'on peut obtenir de l'information sur la rugosité d'une surface à partir de l'étude de la corrélation qui existe entre deux speckles obtenus avec la surface à étudier [6], celle-ci étant éclairée sous deux incidences différentes par deux sources (optique ou acoustique) cohérentes.

Après avoir rappelé les principaux résultats relatifs à la corrélation des speckles, on propose une méthode de mesure de la rugosité basée sur la corrélation de speckles acoustiques. On décrit un interféromètre ultrasonore qui permet de réaliser cette corrélation et on donne des exemples d'analyse de rugosité de surface de béton.

2. Cohérence et corrélation. - 2.1 FORMULE DES INTERFÉRENCES. - Soit l'expérience dite des trous d'Young [8] décrite sur la figure 2.

La source $S$ émet une onde (électromagnétique ou acoustique) $S(t)$. Les trous $S_{1}$ et $S_{2}$ assimilés à des 


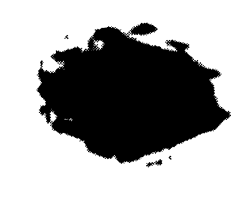

(a)

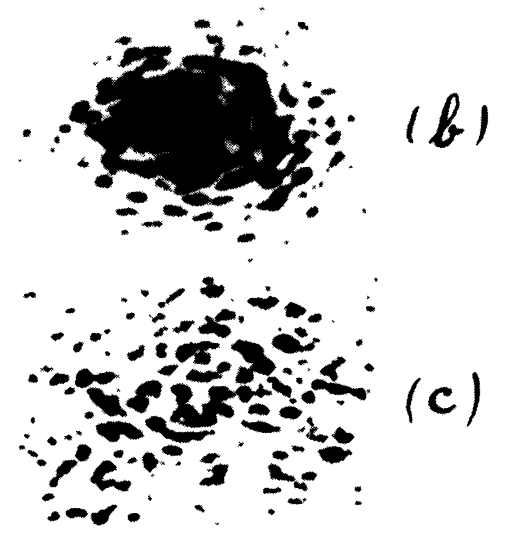

Fig. 1. - Exemples de speckles acoustiques diffusés par des surfaces de rugosités différentes : $(a) \sigma=0,025 \mathrm{~mm},(b) \sigma=0,218 \mathrm{~mm}$, (c) $\sigma=0,664 \mathrm{~mm}$.

[Some examples of acoustical speckles for differents rough surfaces : (a) $\sigma=0.025 \mathrm{~mm},(b) \sigma=0.218 \mathrm{~mm},(c) \sigma=0.664 \mathrm{~mm}$.]

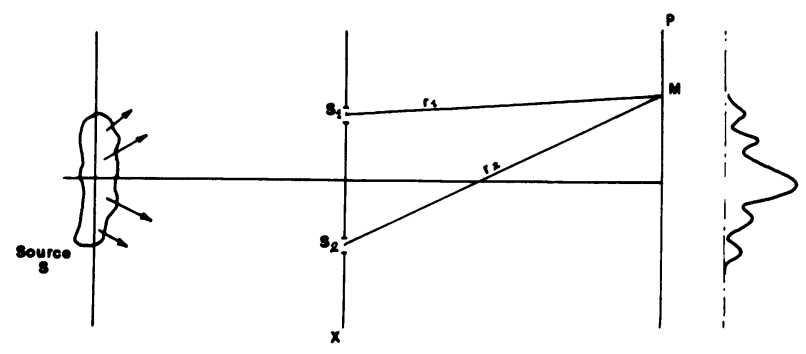

Fig. 2. - Trous de Young.

[Young's fringes experiment.]

systèmes linéaires filtrent $S(t)$. On fait interférer les deux ondes filtrées $S_{1}(t)$ et $S_{2}(t)$ dans un plan P. $\mathrm{Au}$ point $\mathrm{M}$ les interférences liées aux différences de marche $r_{1}$ et $r_{2}$ résultent de la superposition des ondes $S_{1}\left(t-\frac{r_{1}}{c}\right)$ et $S_{2}\left(t-\frac{r_{2}}{c}\right)$. Soit :

$$
S_{\mathrm{M}}=S_{1}\left(t-\frac{r_{1}}{c}\right)+S_{2}\left(t-\frac{r_{2}}{c}\right)
$$

l'intensité $I_{\mathrm{M}}$ est donnée par l'expression

$$
I_{\mathrm{M}}=\left\langle S_{\mathrm{M}}(t) S_{\mathrm{M}}^{*}(t)\right\rangle .
$$

Où $\langle$. $\rangle$ signifie la valeur moyenne. On obtient :

$$
I_{\mathrm{M}}=\left\langle\left|S_{1}\left(t-\frac{r_{1}}{c}\right)+S_{2}\left(t-\frac{r_{2}}{c}\right)\right|^{2}\right\rangle
$$

soit encore :

$$
\begin{aligned}
I_{\mathrm{M}}=I_{1}+I_{2} & +2 \operatorname{Re} \times \\
\times & \left\{\left\langle S_{1}\left(t-\frac{r_{1}}{c}\right) S_{2}^{*}\left(t-\frac{r_{2}}{c}\right)\right\rangle\right\}
\end{aligned}
$$

Re est la partie réelle de la quantité complexe $S$ et $S^{*}$ la quantité complexe conjuguée de $S$.

$I_{1}$ représente l'intensité de l'onde $S_{1}$ au point $\mathrm{M}$, $I_{2}$ représente l'intensité de l'onde $S_{2}$ au point $\mathrm{M}$. A ce fond continu se superpose des phénomènes d'interférences liés à la différence de marche

$$
\tau-\frac{r_{1}}{c}-\frac{r_{2}}{c}
$$

et décrits par l'étude des valeurs moyennes

$$
\Gamma_{12}(\tau)=\left\langle S_{1}\left(t-\frac{r_{1}}{c}\right) S_{2}^{*}\left(t-\frac{r_{2}}{c}\right)\right\rangle
$$

où $\Gamma_{12}(\tau)$ représente la fonction d'intercorrélation entre les deux ondes $S_{1}$ et $S_{2}$. $\Gamma_{12}(\tau)$ peut s'écrire

$$
\Gamma_{12}(\tau)=\left|\Gamma_{12}(0)\right| \exp \left(\mathrm{i} \Phi_{12}\right)
$$

avec

$$
\Phi_{12}=\frac{2 \pi}{\lambda}\left(\left|r_{1}-r_{2}\right|\right)
$$

qui représente la différence de phase introduite par les trajets 1 et 2. Dans ces conditions la relation (2) dite formule des interférences s'écrit :

$$
I_{\mathrm{M}}=I_{1}+I_{2}+2 \sqrt{I_{1} I_{2}}\left|\gamma_{12}\right| \cos \Phi_{12}
$$

après avoir introduit la fonction normée

$$
\gamma_{12}(\tau)=\Gamma_{12}(\tau) /\left[\Gamma_{11}(0) \Gamma_{22}(0)\right]^{1 / 2}
$$

2.2 VisibiLItÉ Des FRANGes. - On définit la visibilité des franges [9] par l'expression :

$$
V=\frac{I_{\max }-I_{\min }}{I_{\max }+I_{\min }}
$$

dans le cas où $I_{1}=I_{2}=I$.

La relation (3) se simplifie

$$
I_{M}=2 I\left(1+\left|\gamma_{12}\right| \cos \Phi_{12}\right)
$$

et il est facile de vérifier que

$$
V=\left|\gamma_{12}\right|
$$

les ondes $S_{1}$ et $S_{2}$ sont cohérentes si $V=1$, elles seront au contraire incohérentes (ou non correlées) si $V=0$. Pour les valeurs intermédiaires de $V$ on dit que les ondes sont partiellement cohérentes.

2.3 CohÉrence en ACOUSTIQUe. - Il est possible de reproduire l'expérience des franges d'Young avec 
des ondes sonores ou ultrasonores. La figure 3 représente le dispositif expérimental dérivé de celui de la figure 2 .

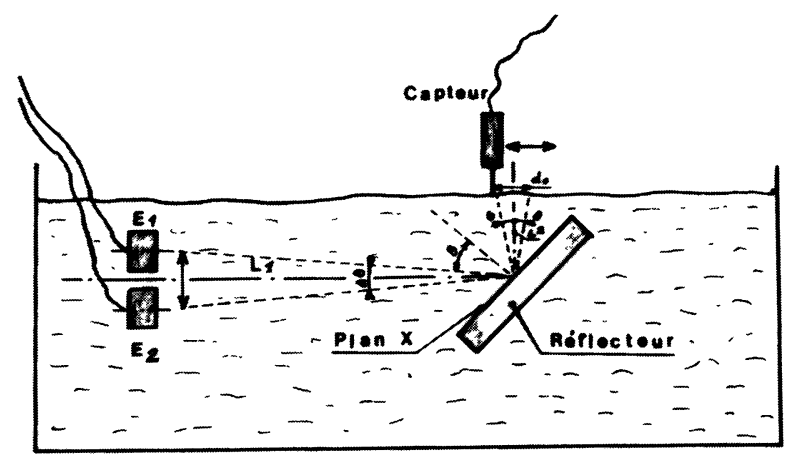

Fig. 3. - Dispositif pour le relevé des franges de Young.

[Experimental arrangement for Young's fringes measurement.]

Les trous $S_{1}$ et $S_{2}$ sont remplacés par deux émetteurs piézoélectriques ultrasonores $\mathrm{E}_{1}$ et $\mathrm{E}_{2}$ fonctionnant à $1 \mathrm{MHz}$. Ils sont séparés par une distance $d=6 \mathrm{~cm}$. Le champ acoustique résultant est enregistré à la surface d'une cuve remplie d'eau, après réflexion sur un réflecteur parfait incliné à $45^{\circ}$. Dans cette première expérience, le réflecteur ne joue aucun rôle si ce n'est de faciliter l'expérimentation. Le capteur piézoélectrique est sensible à la pression ultrasonore. Les distances $L_{1}$ et $L_{2}$ sont respectivement de 120 et $12 \mathrm{~cm}$.

Il en résulte un interfrange donné par

$$
x=\frac{\lambda}{d}\left(L_{1}+L_{2}\right) \sim 3,3 \mathrm{~cm}
$$

où $\lambda / d$ est la séparation angulaire entre franges. La figure 4 montre un exemple de relevé, l'intervalle entre franges est de l'ordre de $30 \mathrm{~mm}$.

3. Corrélation de speckles acoustiques. - 3.1 SUPERPOSITION DE SPECKLES ACOUSTIQUES. - Remplaçons maintenant le réflecteur parfait de la figure 3 par une surface rugueuse. Dans ces conditions chaque émetteur $\mathrm{E}_{1}$ et $\mathrm{E}_{2}$ donne, dans le plan de mesure (surface libre de la cuve acoustique) les speckles d'amplitude $S_{1}$ et $S_{2}$ décalés l'un par rapport à l'autre de la translation :

$$
d_{0}=L_{2} \cos \theta \cdot \Delta \theta .
$$

Si l'écart angulaire $\Delta \theta$ entre les émetteurs $E_{1}$ et $E_{2}$ est petit, on considère que les speckles $S_{1}$ et $S_{2}$ sont identiques au décalage près $d_{0}$ (Fig. 3).

Dans le plan de mesure, l'intensité $I(M)$ sera donnée par la formule (3)

$$
I(M)=2|S|^{2}\left(1+\left|\gamma_{12}\right| \cos \Phi_{12}\right) .
$$

On observera encore des franges d'interférence dont la visibilité dépendra du degré de corrélation entre les deux champs $S_{1}$ et $S_{2}$ diffusés par le réflecteur. Cette

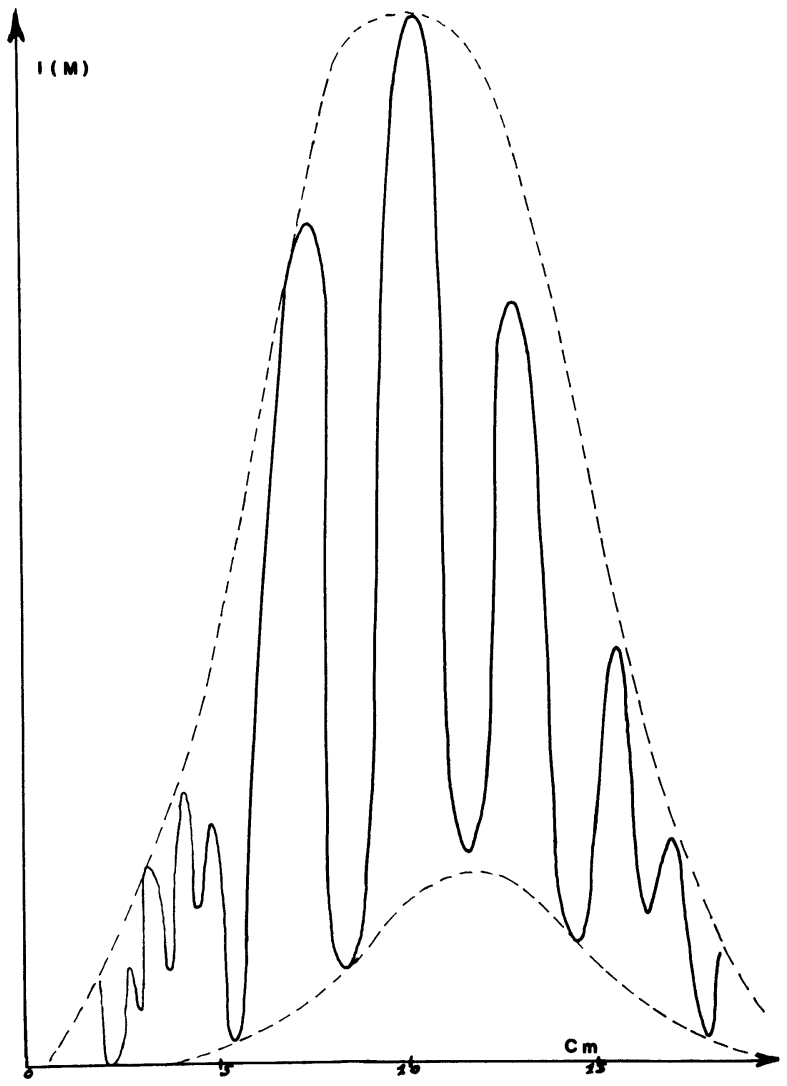

Fig. 4. - Relevé des franges de Young après réflexion sur la surface de référence.

[Young's fringes pattern formed with two beam interference experiment utilizing coherent ultrasonic waves.]

corrélation sera elle-même fonction de la rugosité. La figure 5 montre le profil résultant de la superposition des speckles $S_{1}$ et $S_{2}$.

Ce profil a été obtenu avec le même montage que celui de la figure 3 . Les oscillations rapides que l'on remarque sur l'enregistrement sont liées à la dimension des speckles, les oscillations lentes correspondent aux franges de Young de la figure 4. Les dimensions du capteur (environ $1 \mathrm{~mm}^{2}$ ) sont inférieures aux dimensions des speckles. La période des oscillations rapides correspond à la distance moyenne entre régions voisines d'amplitude maximum ou minimum. Cette dimension est donnée approximativement par [5] :

$$
\varepsilon \sim \frac{1,22 \lambda L_{2}}{D}
$$

où $D$ est le diamètre de la zone diffusante sur le réflecteur rugueux.

Cette zone correspond à la surface couverte par le faisceau incident. Pour un transducteur circulaire de diamètre $\Phi$, elle est donnée par :

$$
D=\frac{1,22 \lambda L_{1}}{\Phi}
$$

d'où

$$
\varepsilon \sim \frac{L_{2}}{L_{1}} \Phi .
$$




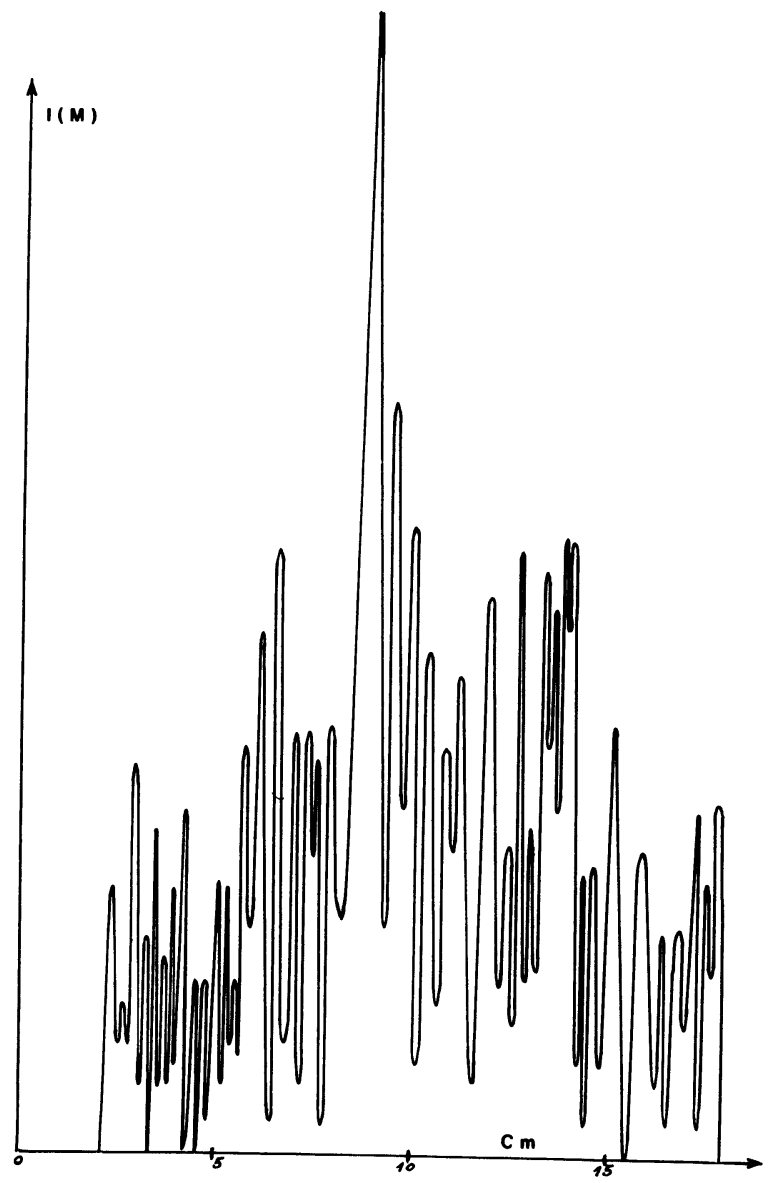

Fig. 5. - Relevé de 2 speckles après réflexion sur une surface rugueuse.

[Young's fringe pattern formed after reflection on a rough surface.]

Pour des transducteurs de diamètre $\Phi=3 \mathrm{~cm}$, la formule (10) donne

$$
\varepsilon \sim 0,3 \mathrm{~cm}
$$

dimension qui correspond bien à celle mesurée sur la figure 5 .

3.2 Calcul du contraste Des franges POUR UNE SURFACE RUGUEUSE. - Il est donc possible d'étudier la rugosité d'une surface à partir de la mesure de la visibilité des franges $V$. Leger et al. [10] ont établi une relation entre la visibilité des franges $V$ et la rugosité de la surface, on va rappeler les principales étapes de ce calcul. Soient $S_{1}\left(\theta_{2}\right)$ le speckle produit par l'émetteur $\mathrm{E}_{1}$ suivant la direction $\theta_{2}$ et $S_{2}\left(\theta_{2}+\delta \theta_{2}\right)$ le speckle produit par l'émetteur $\mathrm{E}_{2}$ suivant la direction $\theta_{2}+\delta \theta_{2}$ (Fig. 6).

Depuis P. Beckman et A. Spizzichino [11] le calcul de l'expression

$$
\left\langle S_{1}\left(\theta_{2}\right) S_{2}^{*}\left(\theta_{2}+\delta \theta_{2}\right)\right\rangle
$$

est classique. Soit une surface s'étendant de $x=-L$ à $x=+L$ l'onde incidente suivant l'angle $\theta_{1}$ issue de

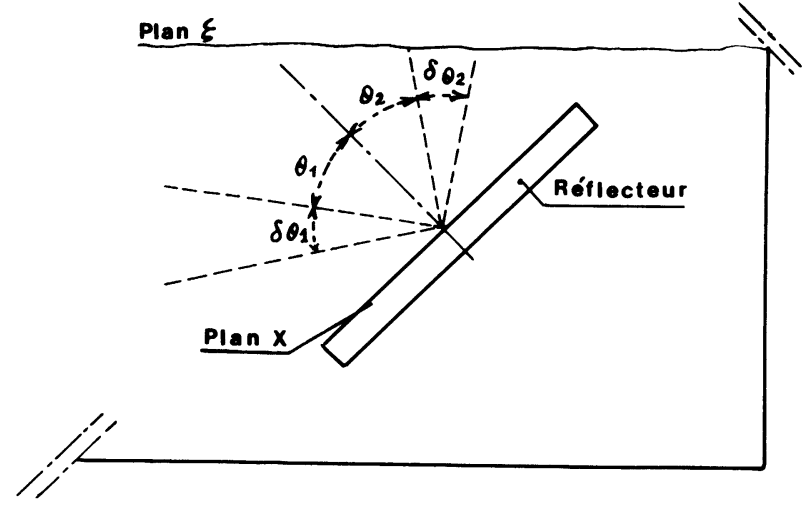

Fig. 6. - Géométrie pour le calcul du contraste des franges.

[Notations used for the visibility of fringes derivation.]

l'émetteur $\mathrm{E}_{1}$ donne une onde réfléchie $S_{1}\left(\theta_{2}\right)$ :

$$
\begin{aligned}
S_{1}\left(\theta_{2}\right)=\frac{A_{0} F_{2}}{2 L} \int_{-L}^{+L} & \times \\
& \times \exp \{i k[a x+\operatorname{ch}(x)]\} \mathrm{d} x_{0}
\end{aligned}
$$

où

$$
\begin{aligned}
& a=\sin \theta_{1}-\sin \theta_{2} \\
& c=-\left(\cos \theta_{1}+\cos \theta_{2}\right)
\end{aligned}
$$

$h(x)$ représente les rugosités de la surface, $A_{0}$ l'amplitude réfléchie suivant la réflexion spéculaire par une surface plane et $F_{2}$ le facteur

$$
F_{2}=\sec \theta_{1} \cdot \frac{1+\cos \left(\theta_{1}+\theta_{2}\right)}{\cos \theta_{1}+\cos \theta_{2}} .
$$

On obtient une expression semblable pour $S_{2}\left(\theta_{2}+\delta \theta_{2}\right)$. On peut alors écrire :

$$
\begin{aligned}
& S_{1}\left(\theta_{2}\right) S_{2}^{*}\left(\theta_{2}+\delta \theta_{2}\right)=\left(\frac{A_{0} F_{2}}{2 L}\right)^{2} \int_{-L}^{+L} \times \\
& \quad \times \exp \left\{i k\left[a_{1} x_{1}+c_{1} h\left(x_{1}\right)\right]\right\} \mathrm{d} x_{1} \\
& \quad \times \int_{-L}^{+L} \exp \left\{-i k\left[a_{2} x_{2}+c_{2} h\left(x_{2}\right)\right]\right\} \mathrm{d} x_{2} .
\end{aligned}
$$

Le calcul de la valeur moyenne [12]

$$
\left\langle S_{1}\left(\theta_{2}\right) S_{2}^{*}\left(\theta_{2}+\delta \theta_{2}\right)\right\rangle
$$

ne porte que sur le facteur aléatoire

$$
\exp \left\{i k\left[c_{1} h\left(x_{1}\right)-c_{2} h\left(x_{2}\right)\right]\right\}
$$

où $h(x)$ correspond aux variations d'amplitude de la surface du réflecteur, par rapport au plan moyen $x$.

Pour aller plus loin dans le calcul, il convient maintenant de formuler des hypothèses relatives aux propriétés statistiques de la surface étudiée.

a) On supposera tout d'abord que les variations d'amplitude $h(x)$ de la surface, donc la rugosité, obéissent à un processus de Laplace-Gauss. Dans ces 
conditions la densité de probabilité des rugosités prend la forme

$$
p(h)=\frac{1}{\sigma \sqrt{2 \pi}} \mathrm{e}-h^{2} / 2 \sigma^{2}
$$

par rapport au plan moyen pris comme plan de référence. $\sigma$ représente la valeur quadratique moyenne des rugosités. Cette valeur sera déterminée expérimentalement par la suite. Avec cette hypothèse, l'expression

$$
\begin{aligned}
\left\langle\operatorname { e x p } \left\{i k \left[ c_{1} h\left(x_{1}\right)-c_{2}\right.\right.\right. & \left.\left.\left.h\left(x_{2}\right)\right]\right\}\right\rangle= \\
& =\Phi\left(k c_{1} h\left(x_{1}\right) ;-k c_{2} h\left(x_{2}\right)\right)
\end{aligned}
$$

qui est la fonction caractéristique associée aux deux groupes de variables aléatoires $k c_{1} h\left(x_{1}\right)$ et $k c_{2} h\left(x_{2}\right)$ est une fonction réelle donnée par

$\Phi=\exp \left\{-\frac{k^{2} \sigma^{2}}{2}\left[c_{1}^{2}+c_{2}^{2}-2 c_{1} c_{2} C\left(x^{\prime}\right)\right]\right\}$

où $C\left(x^{\prime}\right)$ est le coefficient d'autocorrélation spatiale de la surface et $x^{\prime}=x_{1}-x_{2}$.

b) On choisit maintenant une fonction d'autocorrélation du type général :

$$
c(x)=\exp \left(-x^{2} / T^{2}\right)
$$

$T$ représente la distance de corrélation pour laquelle la fonction $C(x)$ prend la valeur $1 / e$.

c) Enfin on considère le paramètre de RayleighBeckmann

$$
g=k \sigma c=\frac{4 \pi \sigma \cos \theta}{\lambda}
$$

qui intervient dans l'expression (14).

Supposons pour simplifier la discussion que $c_{1}=c_{2}$ (ce qui correspond au cas d'un seul émetteur et d'un seul récepteur), alors (14) s'écrit :

$$
\Phi=\exp \left\{-k^{2} \sigma^{2} c^{2}[1-C(x)]\right\} .
$$

Si $x \rightarrow 0, C(x) \rightarrow 1$ donc $\Phi \rightarrow 1$ est maximum, si $x$ augmente $C(x) \rightarrow 0$ et $\Phi \rightarrow \exp \left(-k^{2} c^{2} \sigma^{2}\right)$ qui peut prendre de faibles valeurs si $k c \sigma>1$. Dans ce cas, la valeur de l'intensité ultrasonore moyenne diffusée $\left\langle S_{1}\left(\theta_{2}\right) S_{2}^{*}\left(\theta_{2}+\delta \theta_{2}\right)\right\rangle$ provient presque uniquement des ondes diffusées au voisinage de l'origine pour les faibles valeurs de $x$. On peut alors remplacer la fonction $C(x)$ par les deux premiers termes de son développement, soit :

$$
C(x) \sim 1+x^{2} / T^{2} .
$$

Le tableau I donne les valeurs de $g$ correspondant aux surfaces rugueuses que nous avons étudier. Pour les dalles $F$ et $1, g$ est plus petit que 1 . Pour les autres dalles, $g$ est plus grand que 1 . La dalle de référence sert à étalonner le dispositif, elle est plane avec une valeur de $g$ voisine de zéro. On peut facilement obtenir des valeurs de $g>1$ en augmentant la fréquence de travail. On ne l'a pas fait dans cette étude pour des raisons techniques liées aux transducteurs.

Dans ces conditions [10] on montre que

$$
\begin{aligned}
& \left\langle S_{1}\left(\theta_{2}\right) S_{2}^{*}\left(\theta_{2}+\delta \theta_{2}\right)\right\rangle= \\
& =\left(\frac{A_{0} F_{2}}{2 L}\right)^{2} 2 L \operatorname{sinc}(k \Delta a L) \\
& \times \exp \left(-\frac{\sigma^{2}}{2} k^{2} \Delta c^{2}\right) \frac{T}{\sigma} \sqrt{\frac{\pi}{k c_{1} c_{2}}} \exp \left(-\frac{k^{2} a_{1} T}{4 c_{1} c_{2} \sigma^{2}}\right)
\end{aligned}
$$

avec

$$
\begin{aligned}
\Delta a & =\cos \theta_{1} \delta \theta_{1}-\cos \theta_{2} \delta \theta_{2} \\
\Delta c & =\sin \theta_{1} \delta \theta_{1}+\sin \theta_{2} \delta \theta_{2}
\end{aligned}
$$

et

$$
\operatorname{sinc} x=\sin x / x .
$$

On remarque que dans l'expression (15) seuls les facteurs

$$
\operatorname{sinc}(k \Delta a L) \exp \left(-\frac{\sigma^{2}}{2} k^{2} \Delta c^{2}\right)
$$

dépendent des écarts angulaires $\delta \theta_{1}$ et $\delta \theta_{2}$.

Le premier facteur de (16) est maximum et égal à 1 pour $\Delta a=0$ c'est-à-dire pour

$$
\delta \theta_{2}=\frac{\cos \theta_{1}}{\cos \theta_{2}} \delta \theta_{1} \text {. }
$$

On définit ensuite la visibilité des franges au moyen de l'expression

$\gamma_{12}\left(\delta \theta_{2}\right)=\frac{\left\langle S_{1}\left(\theta_{2}\right) S_{2}^{*}\left(\theta_{2}+\delta \theta_{2}\right)\right\rangle}{\sqrt{\left\langle S_{1}\left(\theta_{2}\right) S_{1}^{*}\left(\theta_{2}\right)\right\rangle\left\langle S_{2}\left(\theta_{2}\right) S_{2}^{*}\left(\theta_{2}\right)\right\rangle}}$.

Soit après calcul et en tenant compte de la condition (17)

$$
V=\left|\gamma_{12}\left(\delta \theta_{2}\right)\right|=\exp \left[-\frac{1}{2}\left(k \frac{\sin \left(\theta_{1}+\theta_{2}\right)}{\cos \theta_{2}} \sigma \delta \theta_{1}\right)^{2}\right]
$$

La formule (19) donne la relation cherchée entre la visibilité des franges et l'écart-type $\sigma$ qui caractérise la rugosité de la surface.

Le résultat (19) n'est valable que si la condition (17) est réalisée, s'il n'en est pas ainsi la fonction $V$ est modulée par la fonction sinc $x$. Dans ce cas on obtient un pic centré sur la valeur de $\theta_{1}$ (Fig. 7). 


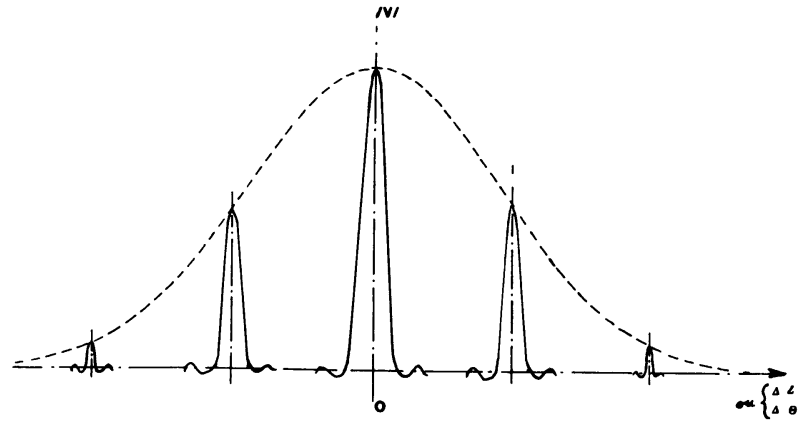

Fig. 7. - Visibilité des franges.

[Visibility of the fringes.]

La fonction $V$ représentée par la formule (19) est une Gaussienne qui décroît d'autant plus vite que la valeur de $\sigma$ est élevée. A partir de la mesure de $V$ on peut calculer la valeur de $\sigma$. En optique cette mesure s'effectue à l'aide d'un interféromètre de Michelson. Il importe de remarquer ici que l'interféromètre de Michelson donne accès à l'expression (18) qui représente la cohérence mutuelle entre des ondes provenant de directions différentes. En acoustique on peut également mesurer $\sigma$ à l'aide d'un interféromètre acoustique que l'on va décrire maintenant.

3. 3 L'INTER FÉROMÈTRE ACOUSTIQUE. - L'interféromètre acoustique a été étudié par C. Bard [13] et s'inspire des interféromètres radioélectriques [14]. La figure 8 donne le schéma de principe de l'interféromètre acoustique.

Il se compose de deux récepteurs piézoélectriques identiques. Les signaux de sortie des deux récepteurs sont amplifiés puis envoyés à l'entrée d'un multiplieur, puis filtrés par un filtre passe bas qui se comporte comme un intégrateur. Soient $S\left(\theta_{2}\right)$ le champ acoustique incident sur le récepteur 1 et $S\left(\theta_{2}+\delta \theta_{2}\right)$ le

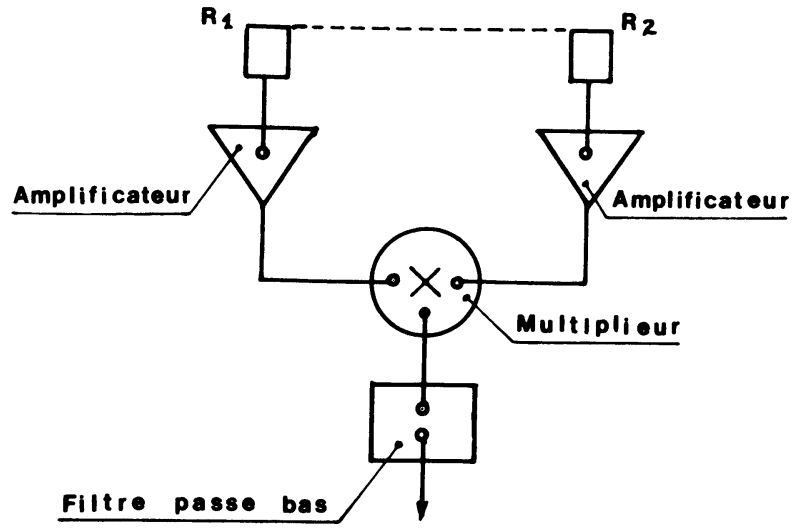

Fig. 8. - Principe de l'interféromètre acoustique.

[Block diagram of the acoustical interferometer.]

champ incident sur le récepteur 2. Dans le cas où la surface rugueuse à étudier diffuse les champs acoustiques émis par les deux émetteurs $E_{1}$ et $E_{2}$, le champ incident sur l'interféromètre sera la somme des champs correspondant à deux speckles décalés, soit

$$
S(\theta)=S_{1}(\theta)+S_{2}(\theta) .
$$

Le récepteur $n^{\circ} 1$ reçoit

$$
S\left(\theta_{2}\right)=S_{1}\left(\theta_{2}\right)+S_{2}\left(\theta_{2}\right) .
$$

Le récepteur $\mathrm{n}^{\circ} 2$ reçoit

$$
S\left(\theta_{2}+\delta \theta_{2}\right)=S_{1}\left(\theta_{2}+\delta \theta_{2}\right)+S_{2}\left(\theta_{2}+\delta \theta_{2}\right) .
$$

Le signal de sortie de l'interféromètre, après multiplication, intégration sera

$\left\langle\left|\left(S_{1}\left(\theta_{2}\right)+S_{2}\left(\theta_{2}\right)\right)\left(S_{1}^{*}\left(\theta_{2}+\delta \theta_{2}\right)+S_{2}^{*}\left(\theta_{2}+\delta \theta_{2}\right)\right)\right|\right\rangle$

et après développement

$$
\begin{aligned}
\left\langle R\left(\theta_{2}\right)\right\rangle=\operatorname{Re}\left\{\left\langle S_{1}\left(\theta_{2}\right) S_{1}^{*}\left(\theta_{2}+\delta \theta_{2}\right)\right\rangle\right. & \left.+\left\langle S_{2}\left(\theta_{2}\right) S_{2}^{*}\left(\theta_{2}+\delta \theta_{2}\right)\right\rangle\right\}+ \\
& +\operatorname{Re}\left\{\left\langle S_{1}\left(\theta_{2}\right) S_{2}^{*}\left(\theta_{2}+\delta \theta_{2}\right)\right\rangle+\left\langle S_{2}\left(\theta_{2}\right) S_{1}^{*}\left(\theta_{2}+\delta \theta_{2}\right)\right\rangle\right\}
\end{aligned}
$$

En se plaçant dans les mêmes conditions expérimentales que D. Leger et J. C. Perrin [10] on montre que le premier terme qui correspond à des champs non correlés est nul. Dans le cas général le deuxième terme est donné par l'expression (15). Si la condition (17) est réalisée, après normalisation, le deuxième terme se simplifie et prend la forme plus simple de l'expression (19).

On se propose maintenant de décrire un dispositif expérimental qui permet d'appliquer les résultats précédents à la mesure des rugosités de surface.

4. Mesure des rugosités de surface. - 4.1 CARACTÉRISTIQUES DES SURFACES ÉTUDIÉES. - On a étudié la rugosité de dalles de béton de $300 \times 200 \times 50 \mathrm{~mm}$. En utilisant des sables de granulométries différentes on a obtenu des rugosités dont l'écart-type $\sigma$ s'étend de 0,025 à $2,39 \mathrm{~mm}$. On a fait une mesure directe de l'écart-type à l'aide d'un comparateur mécanique précis au $1 / 100^{\mathrm{e}}$ de millimètre. La dalle est explorée $\mathrm{cm}$ par $\mathrm{cm}$. Une première série de mesures permet de déterminer la cote du plan moyen de la surface rugueuse.

La rugosité se présente comme une oscillation aléatoire autour du plan moyen. Une deuxième série de mesures permet de calculer l'écart-type de ces oscillations autour du plan moyen.

Le tableau I donne les caractéristiques des surfaces étudiées, écart-type et paramètre $g$ de Rayleigh. 
Tableau I.

$\begin{array}{crrrrrrr}\text { Dalles } & \text { Réf. } & \mathrm{F} & 1 & \mathrm{~N} & 2 & 3 & 4 \\ 2 \sigma \mathrm{mm}\left(10^{-2}\right) & \overline{2,49} & \overline{21,85} & 3 \overline{1,80} & 3 \overline{4,80} & \overline{69,16} & \overline{74,43} & 23 \overline{8,84} \\ g & 0,07 & 0,65 & 0,95 & 1,04 & 2,07 & 2,23 & 7,16\end{array}$

4.2 LE DISPOSITIF EXPÉRIMENTAL. - Avec le dispositif expérimental schématisé sur la figure 9 on étudie la rugosité de surface à l'aide d'une onde ultrasonore de $1074 \mathrm{kHz}$.

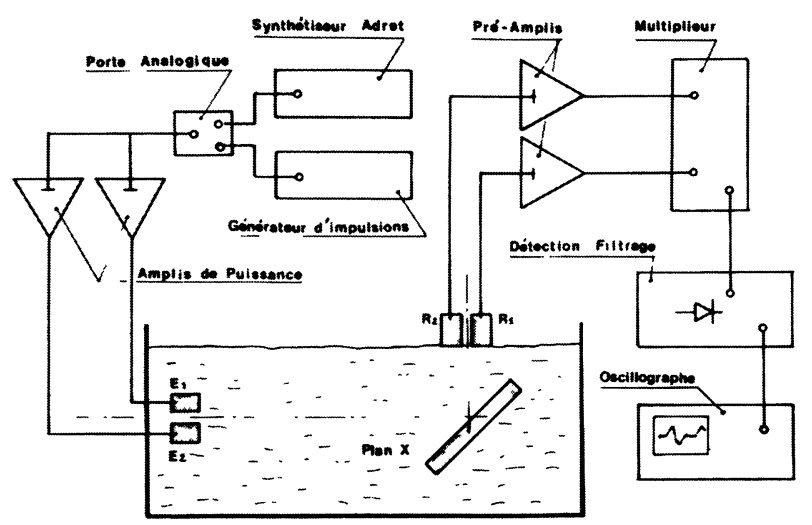

Fig. 9. - Dispositif expérimental pour la mesure des rugosités.

[Experimental set up for measurement of surface roughness.]

A cette fréquence la pénétration des ultrasons dans le béton est insignifiante ce qui évite les réflexions multiples dans l'épaisseur de la dalle. Dans ces conditions, pratiquement toute l'énergie ultrasonore incidente est réfléchie par la face avant.

L'ensemble des émetteurs et récepteurs à ultrasons ainsi que la dalle à mesurer sont entièrement immergés dans l'eau contenue dans une cuve sans écho. Les deux émetteurs identiques de diamètre $30 \mathrm{~mm}$ sont fixés sur un banc vertical l'un au-dessous de l'autre. Un dispositif mécanique permet de les positionner séparément. Ils sont alimentés par dsux amplificateurs de puissance que l'on peut régler de façon indépendante afin d'obtenir deux champs acoustiques identiques en phase et en amplitude. Les signaux émis sont des trains d'onde de $0,2 \mathrm{~ms}$ de durée à la fréquence de $1074 \mathrm{kHz}$. Les champs incidents sont réfléchis par la dalle à mesurer qui est fixée sur un support incliné à $45^{\circ}$. Deux récepteurs ultrasonores identiques de diamètre $30 \mathrm{~mm}$ explorent le champ ultrasonore réfléchi verticalement, Ils sont montés sur un banc horizontal. Un récepteur est fixe, l'autre peut se déplacer. Les tensions de sortie des récepteurs sont appliquées, après amplification, à l'entrée d'un multiplieur. La tension de sortie du multiplieur est détectée puis filtrée, c'est cette tension qui est mesurée et qui donne des valeurs proportionnelles à celles que l'on calculerait à partir de la relation (19).

4.3 MODE OPÉRATOIRE. - Au cours des mesures l'émetteur $E_{1}$ et le récepteur correspondant $R_{1}$
(Fig. 10) sont fixes. Au début des mesures, on met en contact l'émetteur $\mathrm{E}_{1}$ avec l'émetteur $\mathrm{E}_{2}$

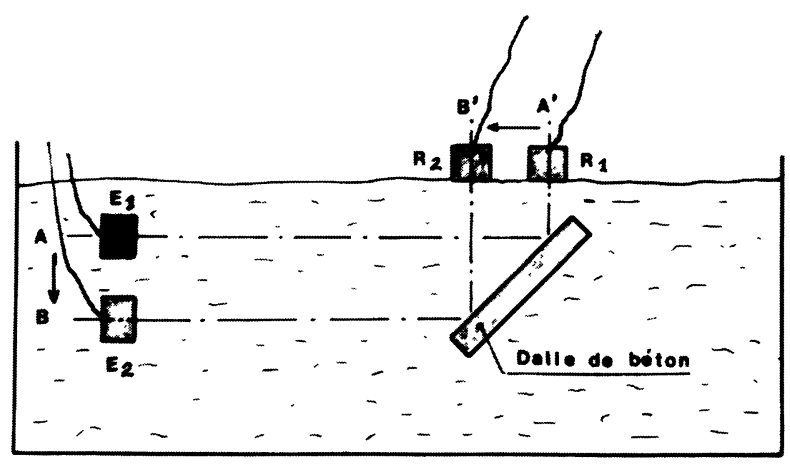

Fig. 10. - Géométrie pour le calcul des angles de réflexion.

[Geometry configurations used in the calculation of the reflection angles.]

On déplace le récepteur $\mathbf{R}_{2}$ afin d'obtenir en sortie du multiplieur un signal maximum. Le point maximum de la courbe 11 correspond au cas idéal où émetteurs et récepteurs seraient confondus. On éloigne ensuite l'émetteur $\mathrm{E}_{2}$ de $\mathrm{A}$ vers $\mathrm{B}$ d'une quantité $\Delta H$, on obtient un nouveau maximum en éloignant le récepteur $\mathrm{R}_{2}$ de $\mathrm{A}^{\prime}$ vers $\mathrm{B}^{\prime}$ d'une quantité $\Delta L$ telle que

$$
\Delta L=\frac{\cos \theta_{1}}{\cos \theta_{2}} \Delta H .
$$

Soit ici par construction du dispositif

$$
\Delta L=\Delta H \quad \text { car } \quad \theta_{1}=\theta_{2}=45^{\circ} .
$$

La relation (21) traduit sur l'appareil la condition (17). Si cette condition n'est pas remplie, les variations d'amplitude du signal de sortie sont proportionnelles aux valeurs de la fonction sinc $x$. Pour chaque valeur du déplacement $\Delta L$ on obtient donc une courbe sinc $x$ dont le maximum décroît lorsque $\Delta L$ croît (Fig. 7). En joignant tous les maxima on obtient la courbe $V$ de la relation (19) tracée en fonction de $\Delta L$. Cette courbe dépend de la rugosité $\sigma$, on obtient donc pour chaque dalle étudiée une courbe $V$.

4.4 RÉSUltats DES MESURES. - Les mesures sont relatives à 4 dalles de rugosité croissantes et les résultats expérimentaux sont portés sur la figure 11.

Pour chaque dalle la courbe $V$ supposée gaussienne a été tracée à partir d'une méthode des moindres carrés. On constate que ces courbes décroissent rapidement quand la valeur de $\sigma$ augmente, la courbe dite de référence correspond à une dalle parfaitement lissée $(\sigma=0,025 \mathrm{~mm})$.

Pour des mesures rapides et moins précises, on peut déterminer l'énergie acoustique diffusée dans le plan des récepteurs. Pour cela, on déplace simultanément les émetteurs et les récepteurs. On fait varier les distances $\Delta L=\Delta H, \mathrm{~cm}$ par $\mathrm{cm}$. Pour une dalle donnée on effectue 11 points de mesure, on calcule ensuite 


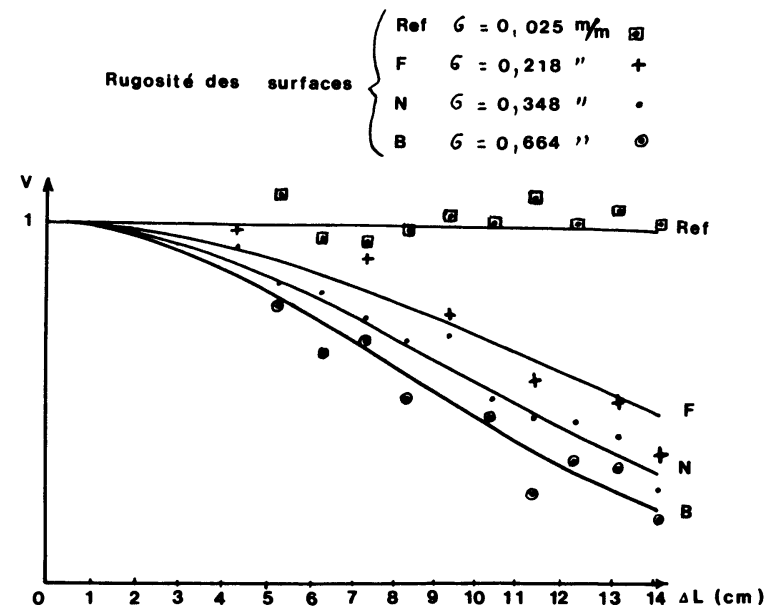

Fig. 11. - Courbes expérimentales de visibilité des franges.

[Experimental cuves for the visibility of fringes.]

l'énergie totale diffusée dans le plan des récepteurs. On peut alors tracer la courbe de la figure 12 donnant les variations de cette intensité en fonction de la rugosité $\sigma$ des surfaces.

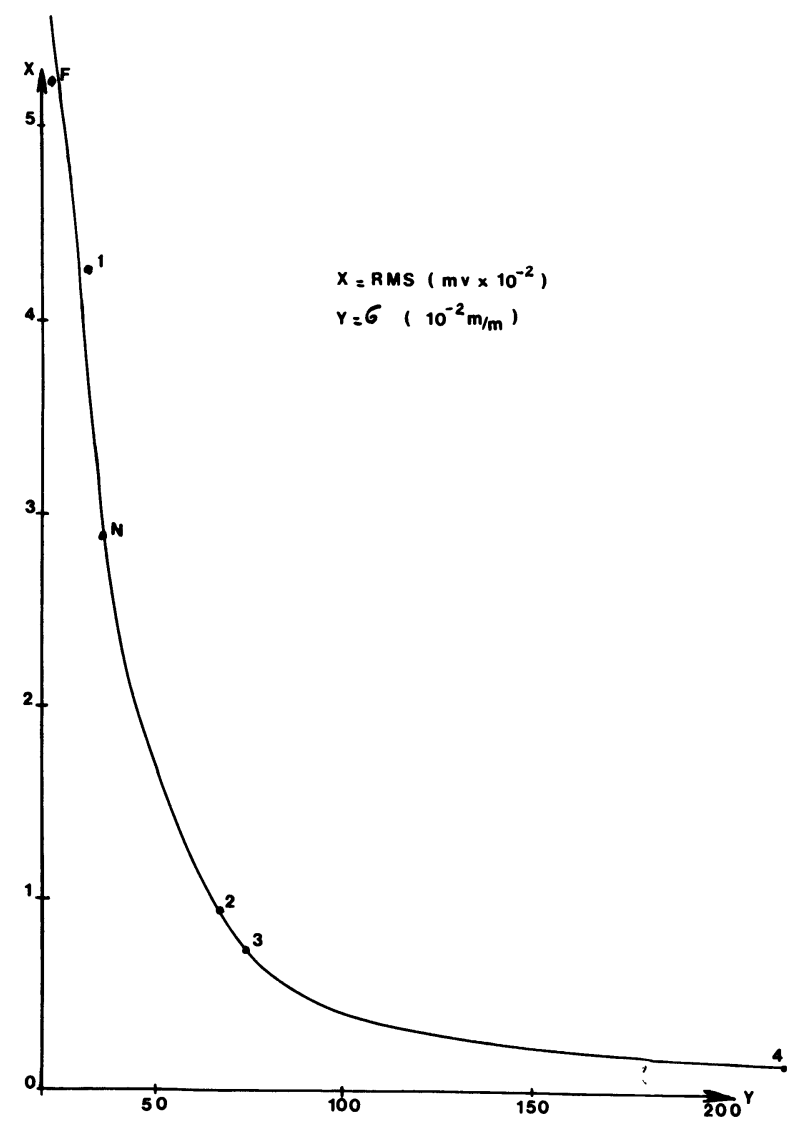

Fig. 12. - Intensité acoustique diffusée en fonction de la rugosité des surfaces.

[Scattered intensity $v s$ surface roughness.]

Cette courbe peut servir de courbe d'étalonnage du dispositif et on peut automatiser le calcul de l'énergie totale diffusée.
4.5 Discussion. - On constate, en observant les courbes de la figure 11, une certaine dispersion des résultats expérimentaux. On peut l'attribuer à l'état des surfaces de béton étudiées. En effet, l'étude directe des rugosités à l'aide d'un comparateur mécanique a fait apparaître les défauts suivants :

- manque de planéité quelquefois important du plan moyen. A cause du retrait, lors de la prise du béton, la surface est concave, pour les dalles 1,2 et $F$, le creux au centre est de l'ordre de 0,$63 ; 1,05$ et $7,12 \mathrm{~mm}$. Ce défaut peut être minimisé en fabriquant des dalles de plus grandes dimensions;

- une distribution granulométrique qui n'est pas isotrope sur la surface. Ce défaut provient essentiellement de la fabrication des dalles.

En effet, pour certaines d'entre elles (dalles 1, 2 et 3), on observe des stries parallèles au grand axe de la surface.

- une distribution d'amplitude qui n'est pas toujours gaussienne. La figure 13 donne un exemple d'histogramme d'amplitude obtenu avec la dalle F.

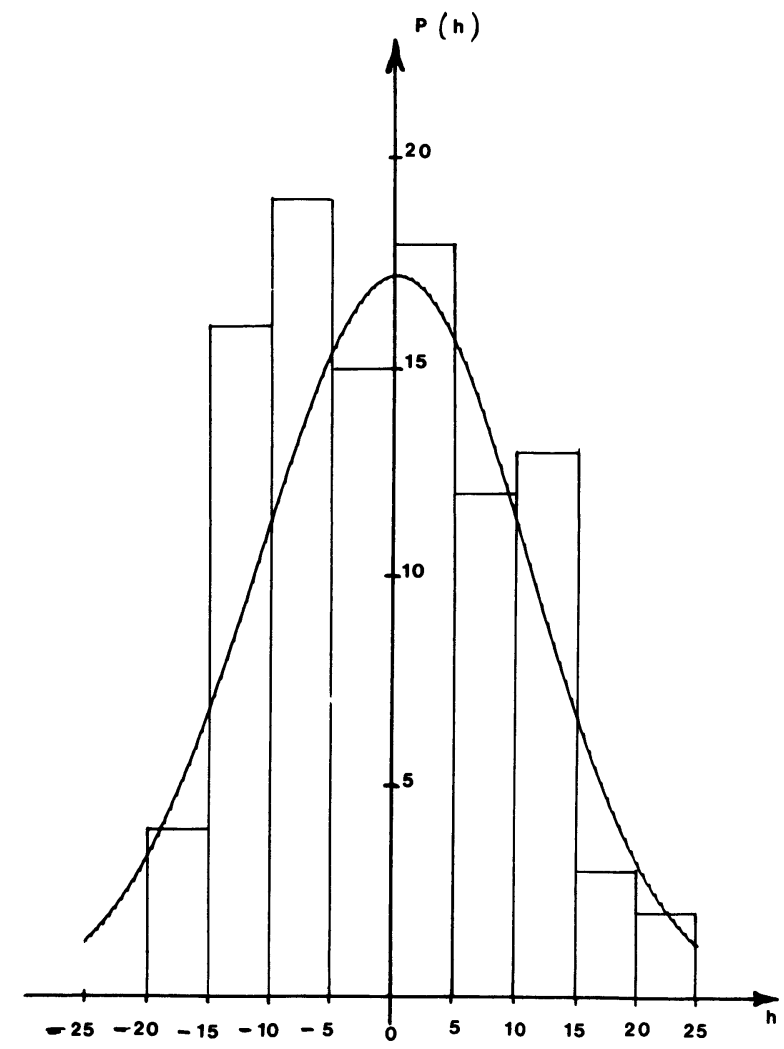

Fig. 13. - Histogramme d'amplitude de la surface F. La courbe représente la loi de probabilité de Gauss.

[Histogram of heights for the F surface. The smooth curve is the Gaussian probability density function.]

On a superposé sur cette figure la densité de probabilité de Gauss de même écart-type : on peut ajuster les histogrammes expérimentaux à d'autres types de densité de probabilité [15] mais alors le calcul de l'intégrale (13) devient rapidement impossible. 
5. Conclusion. - La mesure des rugosités des surfaces par corrélation de speckles a été mise au point en optique. On peut transposer cette technique en acoustique et particulièrement dans la gamme ultrasonore où il est possible de construire un interféromètre acoustique. Les résultats expérimentaux sont en bon accord avec la théorie. Les techniques ultra- sonores prolongent les techniques optiques du côté des mesures des fortes rugosités. Elles peuvent être mise en cuvre dans les liquides opaques (huile, pétrole, milieu marin) et servir au contrôle de la corrosion de réservoirs et d'ouvrages de béton par exemple.

\section{Bibliographie}

[1] Gazanhes, C., Leandre, J. and Lefebvre, J. P., Spectral structure of an ultrasound wave scattered by a random surface : application to the scattering of sound from the sea surface, J. Acoust. Soc. Am. 63 (1978) 1347-1352.

[2] Quentin, G., De Billy, M., Cohen-Tenoudj, F. et JungMAN, A., Caractérisation des surfaces rugueuses par rétrodiffusion ultrasonore, Revue du CETHEDEC 56 (1978) 115-127.

[3] Medwin, H., Bailie, J., Bremhorst, J., Savage, B. J. et ToLstoy, I., The scattered acoustic boundary wave generated by grazing incidence at a slightly rough rigid surface, J. Acoust. Soc. Am. 66 (1979) 1131-1144.

[4] MikesKa, E. E. et MCKinNey, C. M., Range dependence of underwater echoes from randomly rough surfaces, $J$. Acoust. Soc. Am. 63 (1978) 1375-1380.

[5] DainTy EDITOR, J. C., Laser speckle and related phenomena. Topics in applied physics (Springer Verlag, Berlin) 1975.

[6] Francon, M., Granularité laser, speckle, applications en optique (Masson éditeur, Paris) 1978.

[7] Calaora, A., Condat, R., Un perfectionnement de l'imagerie acoustique : l'échantillonnage de phase, Revue d'Acoustique 49 (1979) 121-126.
[8] Blanc-Lapierre, A. et Fortet, R., Théorie des fonctions aléatoires (Masson éditeur, Paris) 1953.

[9] De Velis, J. B. et ReYnolds, G. O., Theory and applications of holography (Addison, Wesley, Co, Reading, Mass.) 1967.

[10] Leger, D. et Perrin, J. C., Real time measurement of surface roughness by correlation of speckle patterns, J. Optic. Soc. Am. 66 (1976) 1210-1217.

[11] Beckmann, P. et Spizzichino, A., The scattering of electromagnetic waves from rough surfaces (Pergamon Press, New York) 1963.

[12] Gazanhes, C., Leandre, J., Propriétés statistiques d'une onde sonore diffusée par une surface aléatoire : application à la réflexion sous-marine de surface, Acustica 30 (1974) 12-19.

[13] BARD, C., Réponse angulaire des interféromètres acoustiques ou radioélectriques à corrélation pour des signaux à large bande, Thèse de Docteur-Ingénieur, Institut Polytechnique de Grenoble (1976).

[14] Swenson, G. W., Mathur, N. C., The interferometer in radio astronomy, Proc. I.E.E.E. 56 (1968) 2114-2130.

[15] Welton, P. J., Frey, H. G. et Moore, P., Experimental measurements of the scattering of acoustic waves by rough surface, J. Acoust. Soc. Am. 52 (1972) 1553-1563. 